\title{
Absence of Pure Nash Equilibria in a class of Co-ordination Games
}

\author{
Chris Cannings ${ }^{1, *}$ and Rob Cannings \\ ${ }^{1}$ School of Mathematics and Statistics, University of Sheffield,UK.
}

Received 14 May 2013; Accepted 20 July 2013

Editor: Vassili Kolokoltsov

\begin{abstract}
We prove that for a certain class of co-ordination games with increasing payoffs for co-ordination there are not necessarily pure Nash equilibria. This is achieved by introducing a particular model for the payoffs. This result is in contrast to the congestion game with decreasing payoff. We discuss a generalization of the model introduced which is of independent interest.
\end{abstract}

Keywords co-ordination; congestion; pure Nash equilibria

DOI: $10.19139 /$ soic.v1i1.10

\section{Introduction}

Situations often arise in which individuals would benefit by co-operating (i.e. choosing the same strategy), but since each would prefer that co-operation to be through the use of a their "own" preferred strategy such co-operation is difficult to achieve. There are various problems associated with such coordination games with respect to how they can be settled so that some convention arises which is acceptable to all players. A recent review [2] discussed various theories regarding the emergence of such conventions, and reports on laboratory experiments designed to test these theories. Our objective here is to demonstrate that even in relatively simple versions of the co-ordination game there may be no pure Nash equilibria so that the issue of convergence of some dynamic model of the game to a co-ordinated equilibrium is not possible.

\footnotetext{
${ }^{*}$ Correspondence to: School of Mathematics and Statistics, University of Sheffield, Sheffield, S3 7RH,UK. Email: c.cannings@ @hef.ac.uk
}

ISSN 2310-5070 Copyright (c) 2013 International Academic Press 
The classical co-ordination game with two players, also called the battle-ofthe-sexes in the symmetric case [1], is often given as an example of a game with more than one pure Nash equilibrium ([4]). Two individuals have personal preferences for one of two different actions, but would also prefer to participate jointly in whatever is chosen. Suppose the individuals are labeled 0 and 1 , and the strategies also as 0 and 1 . If the payoff to individual $i$ when he adopts $j$ and the other player adopts $k$ is $a_{i}(j, k)$, and that $a_{0}(0,0)>a_{0}(1,0), a_{0}(1,1)>a_{0}(0,1)$, $a_{1}(0,0)>a_{0}(0,1)$ and $a_{1}(1,1)>a_{0}(1,0)$, then there are two Nash equilibria, corresponding to the cases where both individuals choose the same action.

When there are more than two players the situation becomes far more complex. [5] recently considered a game in which players receive a payoff which is a monotone increasing function of the number of others who choose the same play. There are $n$ players labeled with the elements of $\mathbf{Z}_{n}$, the integers $\bmod n$, each capable of choosing a pure strategy from $\mathbf{Z}_{k}$. We introduce a notation for the payoffs which is neater for the multi-player case than the obvious generalisations of the "a"-notation used earlier. For a player $i$ there is a function $f_{i}(u, v)$ which is the payoff to the $i$ th player when that player plays $u$ and a total of $v$ players $(v-1$ others) play $u$. The function $f_{i}(u, v)$ is monotone increasing in $v$, for all $i$ and $u$. This was introduced by [5] as a dual to that introduced by [3] in the context of the congestion game with payoff functions $f_{i}(u, v)$ monotone decreasing in $v$.

In [3] it is proved that there is always a pure Nash equilibrium in the congestion game. It is perhaps natural to hypothezise that there will also always be a pure Nash equilibrium in the model of [5]. We demonstrate, by considering a dynamical version of the game, that this is guaranteed only for $k=2$. Specifically we consider the following dynamic version of the game. At each time $t \in\{0,1,2, \ldots\}$ each player chooses to play a particular pure strategy, and then observes their opponent's play. At time $t+1$ they choose the pure strategy which does best against the strategy played by their opponent at time $t$; i.e. they play myopically. We construct a class of examples with $n=k=3$ which do not have any pure Nash equilibria, and use this case to construct examples with no pure Nash equilibria for all $n \geq 3$ and $k \geq 3$.

Finally we consider a class of co-ordination games with $n=k \geq 3$ in which there is an implicit cyclical structure. This allows us to enumerate all the resulting cycles in the dynamical system which correspond to the orbits of the cyclic group action of $C_{2 n}$ on $n$ length binary strings. For $n$ even there are two pure Nash equilibria whereas for $n$ odd there are no pure Nash equilibria. The latter thus provides a further class of co-ordination games with no pure Nash equilibria. 


\section{Multiple players and two strategies}

Suppose $k=2$. If at time $t$ all individuals are playing 1 , then either this is a pure Nash equilibrium or there is some set of players who switch, at time $t+1$, to strategy 0 . This new set of strategies is either a pure Nash equilibrium or there are some players who switch to strategy 0 ; note that those already playing 0 will not switch. We apply this argument recursively and since there are a finite number of players it must terminate, either with some number of players choosing 0 and the remainder (which may be zero) choosing 1, this end point being a pure Nash equilibrium.

\section{A three player, three strategy co-ordination game with no pure Nash equilibrium}

Suppose there are three players and three pure strategies. Again we write $f_{i}(j, x)$ for the payoff to player $i$ when he plays $j$ there being $x$ players using $j$ (including player $i$ ).

Recall $f_{i}(u, v)$ is monotone increasing in $v$. We consider three conditions on the payoff functions $f_{i}(u, v)$. Note that numbering for players and for strategies are all done modulus 3 .

Now,

(1) Player $i$ prefers strategies $i$ and $i+1$ to $i+2$ or equivalently $f_{i}(i+2,3)<$ $f_{i}(i, 1), f_{i}(i+1,1)$.

(2) Player $i$ prefers strategy $i$ if another player plays $i$ or equivalently $f_{i}(i+$ $1,3)<f_{i}(i, 2)$.

(3) Player $i$ prefers strategy $i+1$ if no other player plays $i$ or equivalently $f_{i}(i, 1)<f_{i}(i+1,1)$.

For a coordination game, these three conditions are equivalent to the following chain of inequalities:

$$
f_{i}(i+2,1) \leq f_{i}(i+2,2) \leq f_{i}(i+2,3)<f_{i}(i, 1)<f_{i}(i+1,1) \leq
$$

$f_{i}(i+1,2) \leq f_{i}(i+1,3)<f_{i}(i, 2) \leq f_{i}(i, 3) \quad i=0,1,2$.

Suppose from now on that these conditions hold for the payoff functions. We shall show that there is only ever a single Nash equilibrium and that each player plays a mixed strategy. In particular, there is no Nash equilibrium in pure strategies.

Suppose in some Nash equilibrium that player 0 always plays strategy 0 . Now player 2 can only play strategy 0 or 2 , i.e. not 1 , and so since player 0 is not playing strategy 1 (3), player 1 will play strategy 2 exclusively. Then by condition (2), player 2 will also play strategy 2 exclusively. This however is not a Nash equilibrium as player 0 is motivated to switch to strategy 1 by condition (3). Thus by contradiction, no Nash equilibrium has player 0 playing strategy 0 exclusively. 
Suppose then that a Nash equilibrium has player 0 always playing strategy 1 . In this case the conditions mean that player 1 will play strategy 1 and player 2 will play strategy 0 exclusively. Again this is not a Nash equilibrium since player 0 is motivated to switch strategy. Thus player 0 plays a mixed strategy in any Nash equilibrium. Similarly players 1 and 2 must also play mixed strategies in any Nash equilibrium.

We can construct a dynamical system in discrete time where at any moment in time the state consists of a pure strategy for each player and where the successor state has each player (myopically) choosing the strategy on the assumption that the other players will stick with their current strategies. A state is a fixed point of the dynamic if, and only if, it is a pure Nash equilibrium.

This implies there are eight possibilities $\{0,1\} \times\{1,2\} \times\{0,1\}$ where $\times$ denotes the cartesian product. These are all ruled out as pure Nash equilibria. Consider the digraph $\mathbf{G}=\{\mathbf{V}, \mathbf{E}\}$, where the vertices $\mathbf{V}=\{(u, v, w) ; u, v, w \in$ $\{0,1,2\}\}$, and $\mathbf{E}$ contains an edge $x \rightarrow y$ if $y$ is the outcome of myopic plays by the individuals when in state $x$. This captures the dynamics of the system under myopic play, and there are 27 states, 19 of which are transient, there is a two cycle $(0,1,2) \leftrightarrow(1,2,0)$, in which at each stage all players switch their strategy, and a six cycle $(\mathbf{1}, 1,0) \rightarrow(0, \mathbf{1}, 0) \rightarrow(0,2, \mathbf{0}) \rightarrow(\mathbf{0}, 2,2) \rightarrow(1, \mathbf{2}, 2) \rightarrow(1,1, \mathbf{2})$, in which one player (indicated in bold type) switches its strategy at each stage .

There will be a unique Nash equilibrium and it involves all eight of the states $\{0,1\} \times\{1,2\} \times\{0,1\}$. Suppose now that the functions $f_{i}(i+w, v)$ are independent of $i$, which we refer to as the symmetric case (though the game is not symmetric). In order to simplify the notation we define $a=f_{i}(i, 1), b=$ $f_{i}(i, 2), c=f_{i}(i+1,2)$ and $d=f_{i}(i+1,1)$, so that $a<d<c<b$. The payoff to player 0 when he plays the three strategies with probabilities $\left(p_{0}, p_{1}, 0\right)$, player 1 plays $\left(0, q_{1}, q_{2}\right)$, and player 2 plays $\left(r_{0}, 0, r_{2}\right)$ is $\left(p_{0}\left[a r_{2}+b r_{0}\right]+p_{1}\left[c q_{1}+d q_{2}\right]\right)$. Thus we require for a Nash equilibrium the indifference condition $\left[a r_{2}+b r_{0}\right]=$ $\left[c q_{1}+d q_{2}\right]$ and so $(c-d) q_{1}+(b-a) r_{2}=(b-d)$. Each of the terms in brackets is positive. Similar expressions arise for the other two players. We observe that the equilibrium must necessarily be unique because of the linearity of the indifference condition, and so $p_{0}=q_{1}=r_{2}$ and $p_{1}=q_{2}=r_{0}$. Substituting, in terms of the $p_{0}$ and $p_{1}$ we obtain condition $\left(a p_{0}+b p_{1}\right)=\left(c p_{0}+d p_{1}\right)$. So, $\frac{p_{0}}{1-p_{0}}=\frac{(b-d)}{(c-a)}$ giving $p_{0}=\frac{(b-d)}{(c-d)+(b-a)}$.

We note in passing that if we retain the dependence of the payoffs on the player then it is still relatively straightforward to find the unique Nash equilibrium.

\subsection{Counterexample for $n \geq 3$ and $k \geq 3$}

Having constructed a co-ordination game with no pure Nash equilibrium for $n=3$ and $k=3$ we use this to give counterexamples for all $n>3$ and $k \geq 3$. Begin by choosing payoff functions so that players $\{3,4, \ldots, n\}$ always play 0 , 
players 1 and 2 have the same preferences as before while player 0 plays 1 if $n-3$ players play 0 , and 1 otherwise. Essentially then the behaviour of players $\{3,4, \ldots, n\}$ does not alter the behaviour of players 0,1 and 2 vis-a-vis the $n=k=3$ counterexample, and so there is no pure Nash equilibrium, and for $i \in\{0,1,2\}$ player $i$ plays strategy $i$ with the same probability as in the $n=k=3$ case.

\section{A generalised model with $n=k$}

The example constructed for $n=k=3$ can be easily generalised to the case where we have $n=k \geq 3$. We use the same set of preferences for the players, as given for the $n=k=3$ case.

The inequalities are now

$f_{i}(i+v, 1) \leq f_{i}(i+v, 2) \leq f_{i}(i+v, u)<f_{i}(i, 1)<f_{i}(i+1,1) \leq$ $f_{i}(i+1,2) \leq f_{i}(i+1, u)<f_{i}(i, 2) \leq f_{i}(i, u)$

$i \in \mathbf{Z}_{n}, u \geq 3, v \in \mathbf{Z}_{n} \backslash\{0,1\}$

This guarantees that player $i$ can only play $i$ or $i+1$ in a Nash equilibrium.

A neat way to study the process is to use a binary representation. A state is represented as a binary cycle of length $n$, where the $i$ th entry is 0 if the $i$ th individual is playing strategy $i$, and is 1 otherwise. We write these as a linear string but the final element is considered adjacent to the first.

Now the transition under the payoffs specified is from state $u$ to state $C_{n}^{*}(u)=$ $C_{n}(\bar{u})$ where $C_{n}$ is an operator which moves each element forward one position (mod $\mathrm{n}$ ), and $\bar{u}$ is obtained from $u$ by changing 0 's to 1's and changing 1's to 0's.

Example $(00011) \rightarrow(01110)$.

It follows immediately that the number of changes at each step is constant and equal to the number of adjacent pairs in $u$ which are equal. Pure Nash equilibrium are precisely those states which do not change under the dynamic; in other words precisely those where there are no adjacent pairs which are equal. Such a state has alterating 0's and 1's so cannot occur for $n$ odd. Given a sequence of bits $B$ we write $B^{r}$ for the sequence in which $B$ is repeated $r$ times. For $n=2 m$ the states $(01)^{m}$ and $(10)^{m}$ are (the only) pure Nash equilibria.

We have the group $C_{2 n}$ acting on the set of $n$ length binary strings in which the canonical generator acts as $C_{n}^{*}$, the cycles corresponding to the orbits. This situation is often described as corresponding to a necklace, and the question asked is how many permutationally distinct necklaces of $n$ beads can be made when we have beads of $c$ colours. Here we have 2 colours ( 0 and 1$)$, the necklace is envisaged to be lying on a table and cannot be turned over, and we have one additional unusual equivalence since switching 0's and 1's does not produce a different necklace. 
We can specify all the cycles for some $n$ by listing the lexicographically earliest member ( 0 precedes 1 ) of the permutationally (under $C_{n}^{*}$ ) distinct states with at least as many 0's as 1's. We give some examples and include the number of changes associated with each step in the cycle. For $n$ prime there is one two cycle and all other cycles are of length $2 n$.

Example $n=k=5$ then we have states $(0)^{5}, 00001,00011$ and 00101 which initiate respectively cycles of length 2 with 5 changes, two with length 10 and 3 changes and one with length 10 and 1 change.

Example $n=k=9$. We have states $(0)^{9}$ with cycle length 2 and 9 changes, $(001)^{3}$ with cycle length 6 and 3 changes , and states

(000000001), (000000011), (000000101), (000001001), (000010001), (000000111), (000001011), (000001101), (000010011), (000011001), (000100011), etc, with the number of changes being $7,7,5,5,5,7,5,5,5,5,5$ respectively, there being 28 of these with cycle lengths 18 .

Example $n=k=15$. States $(0)^{15}$ cycle length 2 with 15 changes, $(001)^{5}$ cycle length 6 with 5 changes, $(00001)^{3},(00011)^{3},(00101)^{3}$ of length 10 and 9 changes, and 1091 cycles of length 30 .

Note that each initial state, other than that consisting of all 0's, being the earliest in lexicographic order starts with a 0 and ends with a 1 . It therefore consists of an even number of runs, where a run refers to a sequence of identical bits. Suppose these runs have lengths $x_{i}$ for $i=1, \ldots, 2 h$. Then the number of changes, equal to the number of consecutive pairs of 0's and 1's, is $\Sigma_{i=1,2 h}\left(x_{i}-1\right)=n-2 h$ which is odd. It is clear that for any odd $n$ we can have cycles with all possible odd numbers of changes between 1 and $n-2$, plus that starting with all 0 's which has cycle length 2 and number of changes $n$.

The unique Nash equilibrium is easily derived and in fact the probability that a player $i$ chooses strategy $i$ is again $\frac{(b-d)}{[(c-d)+(b-a)]}$ in the symmetric case.

\section{Discussion}

Congestion games with payoffs monotone decreasing with the number of others using the same play always have pure Nash equilibria. In contrast we demonstrate in this paper that in co-ordination games with payoffs monotone increasing with the number of others using the same strategy, a natural dual of the congestion game, there may not exist a pure Nash equilibrium.

Our demonstration is built around a case with three players and three strategies. From this simple example, which has no pure Nash equilibrium, we construct examples for all numbers of players and number of strategies.

As a secondary aspect of the paper we generalize this example to cases where there are equal numbers of players and strategies. Underlying this model, which we believe has intrinsic interest, is a group whose orbits are the cycles which occur 
in the game, and the notion of a necklace, though with an unusual extra condition that a necklace and its complement with respect to colour are equivalent. It also corresponds to a finite automata on a directed circle where each individual updates to the value currently played by the neighbour to which its out-edge points.

\section{Acknowledgement}

We thank Dr Richard Southwell for bringing this problem to our attention and acknowledge support for $\mathrm{CC}$ from the European Union through funding under FP7-ICT-2011-8 project HIERATIC (316705).

\section{REFERENCES}

1. K. Binmore, Fun and Games: A Text on Game Theory, (1992), D.C.Heath, Lexington, Mass.,USA.

2. V. Frey, R. Cortens \& V. Buskens, Co-ordination Games: Equilibrium selection in network co-ordination games: an experimental study, Review of Network Economics, 11 (2012), pp. $1-26$.

3. I. Milchtaich, Congestion games with player-specific payoff functions, Games and Economic Behaviour, 13 (1996), pp. 111-124.

4. J. Nash, Non cooperative games, Annals of Mathematics, 54 (1951), pp. 286-295.

5. R. Southwell, J. Huang \& B. Shou, Graphical Games with Applications to Social, Economic and Wireless Networks, (2013), In Preparation. 